\title{
Characteristics and evaluation of urban soundscapes worthy of preservation
}

\author{
Yihong Jia ${ }^{\text {a, Hui Ma }}{ }^{\mathrm{a}, *}$, Jian Kang ${ }^{\mathrm{a}, \mathrm{b}}$ \\ a School of Architecture, Tianjin University, Tianjin, 300072, China \\ ${ }^{\mathrm{b}}$ Institute for Environmental Design and Engineering, University College London, UK
}

\begin{abstract}
Improvement of the sound environment is essential for improving the overall quality of the urban environment and for reflecting the characteristics of a city. Unfortunately, some soundscapes in cities are gradually being destroyed by the rapid development. Therefore, it would appear that the preservation of soundscapes is as necessary and crucial as noise control in cities. In this paper, two field studies were carried out at two stages to explore the types and characteristics of urban soundscapes that should be preserved, based on the example of Tianjin, China. At stage one, 2504 respondents participated in social surveys to recommend the soundscapes worthy of preservation in the city. At stage two, a group soundscape walk with twenty-three participants was carried out to explore the characteristics of soundscapes worthy of preservation suggested by the respondents at the first stage. The following results were obtained: (1) regarding the attitude towards the urgency of soundscape preservation, $79 \%$ of the respondents thought it was urgent or very urgent, with an average score of 4.04 (five-point numeric scale was used and five meant very urgent). (2) The majority of soundscapes worthy of preservation were located in urban parks and historic districts with respective proportions of $66 \%$ and $20 \%$. The top three sound source categories of these soundscapes were social/communal sounds (talking, bells, laughter, and sounds from human
\end{abstract}


activities), animal sounds (birdsong and sounds from non-domesticated animals) and water sounds. (3) Relaxation, vibrancy, representativeness, strength, and richness were principal characteristics of the soundscapes worthy of preservation. (4) Based on their characteristics, these soundscapes were classified into five clusters using hierarchical cluster analysis (HCA). They were clusters of relaxing nature soundscape, vibrant nature soundscape, vibrant human activity soundscape, culture-related soundscape and traditional soundscape. The dominant characteristic of each cluster was driven by its sound mark rather than the dominant sound.

Keywords: Urban soundscapes, Preservation, Characteristics, Classification 


\section{Introduction}

Improving the sound environment is an essential way to improve the overall urban environmental quality, and to reflect the characteristics of a city (de la Prida et al., 2019; Kang, 2006). However, with the rapid process of urbanisation, the urban sound environment is deteriorating (Hong and Jeon, 2014; Iglesias et al., 2014; Quintero et al., 2018). For example, noise complaints in China accounted for $39.2 \%$ of the total number of environmental complaints in 2013, and it increased to 50\% in 2017 (Han et al., 2018). In addition to the efforts of noise control, some soundscapes can also contribute positively to mitigating noise problems (Pijanowski and Farina, 2011; Van Renterghem, 2019; Zhang et al., 2018). Besides this benefit, the soundscapes also have other ecological and social values (Irvine et al., 2009). The natural soundscapes have positive effects on human health (Aletta et al., 2018), and the cultural soundscapes could be considered as part of the cultural heritage (Kato, 2009). Therefore, soundscape preservation should be considered in order to improve the urban sound environment.

Those associated values of soundscapes referred above strengthen the motivation of soundscape preservation, thus numerous projects and investigations have been performed to identify the soundscapes worthy of preservation. In the 1960s, Schafer founded "World Soundscape Project (WSP)" to educate residents to appreciate and preserve the sound around them from aesthetic and cultural perspective. Schafer also explained that the sound mark as the key component of soundscape was the most representative sound that symbolized the certain place or time, thus preserving the sound mark should be an important object in urban planning or landscape 
design (Schafer, 1993). Influenced by the WSP, Finland launched "100 Finnish soundscapes", a three-year project to select the important soundscapes in Finland (Liu and Kang, 2016). Later, from 1994 to 1997, the Japanese Environmental Agency carried out a project named "100 Soundscapes of Japan: Preserving Our Heritage" to investigate the soundscapes worthy of preservation throughout Japan (Hiramatsu, 2006; Torigoe, 2003). A similar project named "100 beautiful sounds of Korea-1999" was carried out in Korea (Han and Oh, 2008) to practice the ideology of soundscape. Recently, Turkey made its first attempt to collect cultural sound in Istanbul (Yelmi, 2016). In addition to these surveys conducted on a relatively large scale, some projects were performed in specific small blocks, such as the national parks (Miller, 2008) or other nature reserves (Francis et al., 2017; Furumo and Aide, 2019; Weinzimmer et al., 2014), historic city centres (Alazaizeh et al., 2019; Huang and Kang, 2015) and small villages (Järviluoma, 2000). These existing investigations have provided a detailed summary of the soundscapes worthy of preservation from both the entire countries and the specific small blocks. However, the urban soundscapes in cities have their own characteristics and internal relationship, additional studies on soundscape preservation from the perspective of city level also need to be conducted to create more active and vigorous cities.

Furthermore, to formulate the reasonable preservation policy, the characteristics of soundscapes worthy of preservation need to be analysed. There are two types of studies investigating the characteristics of soundscapes. One type focussed on the soundscapes in specific areas, such as urban parks (Liu et al., 2018a; Ou et al., 2017), residential areas (Rey and Barrigon, 2017), commercial streets (Yu et al., 2016) and urban waterfront areas (Puyana et al., 2016). The 
other investigated particular soundscapes, such as water sounds and birdsongs (Liu et al., 2019; Zhao et al., 2018). However, studies on the characteristics from the viewpoint of soundscapes worthy of preservation are still lacking. Moreover, a reasonable classification of the soundscapes worthy of preservation can improve the efficiency of the preservation work. A previous study has suggested eight clusters according to the ecological and social values of soundscapes (Dumyahn and Pijanowski, 2011). However, this classification did not consider the perceived characteristics of soundscapes. Therefore, more research is still required to create a comprehensive classification of soundscapes worthy of preservation.

The aim of the present study is to understand the characteristics of soundscapes worthy of preservation and provide practical suggestions on preservation management. Specifically, the study aims to explore (1) the basic attitude of respondents towards soundscape preservation, (2) the urban soundscapes worthy of preservation at the city scale, (3) the characteristics of the preservative soundscapes, and (4) the categorisation of urban soundscapes worthy of preservation based on the perceived characteristics.

\section{Methodology}

In this paper, two field studies were carried out at two stages to explore the types and characteristics of urban soundscapes that should be preserved. At stage one, social survey was conducted to investigate what kind of soundscapes were worth preserving. At stage two, a group soundscape walk with twenty-three participants was carried out to explore the characteristics of 
the preservative soundscapes selected according to the results of the first stage.

\subsection{Survey site selection}

The study was conducted in Tianjin, a typical city in Northern China, which has an abundance of natural resources, such as mountains, rivers, and the Yellow Sea. Haihe River, as the mother river of Tianjin, traverses the city from the North to the South and forms a unique riverside landscape, which attracts a large number of local citizens and tourists. Furthermore, during more than 600-years of development history, Tianjin has accumulated rich historic and cultural resources. At present, there are twelve blocks and nearly 800 buildings with historic characteristics, which are of great value. Abundant types of soundscapes have been formed in Tianjin owing to the profound cultural and historic character as well as the beautiful scenery (Marinelli, 2010). However, as one of the municipalities in China, the sound environment in Tianjin has changed a lot in recent years with the rapid development. More noise problems emerged and some valuable soundscapes disappeared. How to preserve urban soundscapes effectively and scientifically is the problem that should be considered by Tianjin and other rapid developing cities.

\subsection{Stage one: data collection of social survey}

In order to investigate what kind of soundscapes should be preserved, both face-to-face and online surveys were conducted. Face-to-face surveys were conducted from 6:00 to 19:00 every other day from June to December in 2016 in six districts and four suburbs of Tianjin to obtain a 
wide range of opinions across the whole city.

In total, three groups of population participated in this survey, including: (1) local residents, who had lived in Tianjin for more than three years. They were familiar with the sound environment of Tianjin and their opinion could reflect both current perception and memory of the sound environment. (2) Tourists, who had lived in Tianjin for less than two weeks. Their opinion might reflect the most impressive and attractive aspects of the sound environment in Tianjin. (3) Landscape architects and urban planners, whose responses represented the demand of urban soundscape from the professional perspective. Their responses were collected through snowball sampling by WeChat network. Finally, 2504 valid questionnaires were collected and 162 questionnaires were excluded because more than $20 \%$ of the questions in the questionnaire were not answered. The demographic information of all the respondents is listed in Table 1. It can be observed that the ratio of male to female was 1.03: 1, which was similar to the proportion of the residents in Tianjin (Liu et al., 2018b), and most of the respondents had received advanced education, possibly because the respondents with high education level were more interested in our study.

The questionnaire used in the social survey contained two sections: (1) people's attitude towards urban soundscape preservation, including their cognition on the importance of soundscapes in improving the urban environment, their attitude towards soundscape deterioration, and the urgency of soundscape preservation. Five-point verbal scale from not at all to extremely was used for each question. (2) The soundscapes worthy of preservation based on respondents' personal experience. In this section, respondents were asked to recommend soundscapes freely 
based on their experience of the whole city instead of the limited living and activity areas using an open question. In addition, in the guidance section at the beginning of the questionnaire, the concept of soundscape and some soundscape examples were given to help the respondents understand this study.

Table 1 Demographic information of the respondents

\begin{tabular}{ccccc}
\hline & Tourist & Resident & $\begin{array}{c}\text { Urban planner/ } \\
\text { Landscape architect }\end{array}$ \\
\hline Total & Male & $6.04 \%$ & $87.49 \%$ & $6.47 \%$ \\
\hline \multirow{2}{*}{ Gender } & Female & $41.90 \%$ & $50.71 \%$ & $51.65 \%$ \\
& $<20$ & $7.29 \%$ & $5.91 \%$ & $48.35 \%$ \\
\hline \multirow{3}{*}{ Age groups } & $20-29$ & $51.25 \%$ & $46.45 \%$ & 0 \\
& $30-39$ & $19.58 \%$ & $12.50 \%$ & $59.34 \%$ \\
& $40-49$ & $10.75 \%$ & $5.07 \%$ & $25.27 \%$ \\
& $50-59$ & $5.76 \%$ & $3.16 \%$ & $13.19 \%$ \\
Occupation & $>60$ & $5.37 \%$ & $2.48 \%$ & $2.20 \%$ \\
& Student & $66.45 \%$ & $57.95 \%$ & 0 \\
\hline \multirow{2}{*}{ Education level } & Pensioner & $13.82 \%$ & $4.29 \%$ & 0 \\
& Working person & $19.74 \%$ & $63.84 \%$ & 0 \\
& Primary & $4.62 \%$ & $1.86 \%$ & $00 \%$ \\
\hline & Secondary & $24.08 \%$ & $19.00 \%$ & 0 \\
& University & $52.02 \%$ & $59.58 \%$ & $24.53 \%$ \\
\hline
\end{tabular}

\subsection{Stage two: data collection of group soundscape walk}

At stage two, a group soundscape walk was performed to explore the perceived characteristics of the soundscapes worthy of preservation. It is widely known that the perceptions of soundscapes are closely related to their visual environment (Pérez-Martínez et al., 2018; Zhao et al., 2018). Therefore, in order to provide an in-situ experience of the soundscapes, a group soundscape walk 
was carried out from 9 am to 5 pm on March 27, 2017. Besides, compared to survey methods, the advantage of soundscape walk is the relatively small sample size (Jeon and Hong, 2015). Recent studies using the method of soundscape walk have attached importance to the opinions of experts on soundscapes or urban planning in order to apply the results to real-life scenarios through urban planning and design (Jeon et al., 2013). Thus, twenty-three graduate students from the School of Architecture, Tianjin University were recruited to assess the soundscapes along the route planned by the researcher. All of them have research and design experience on urban planning or landscape design. Among them 14 males and 9 females aged from 20 to 25. All the participants reported that they had normal hearing and vision. In order that these participants could thoroughly understand the investigation process, a pilot training was conducted for them.

The group soundscape walk was carried out in five locations in Tianjin, where there were twelve soundscapes recommended to be preserved more than twenty times in the social survey. Fig. 1 shows the twelve soundscapes of this group soundscape walk, which were selected via the following steps. Firstly, according to the result of the social survey, the soundscapes recommended more than twenty times were classified into six categories based on the method proposed by Brown (Brown et al., 2011a). Secondly, considering the following three factors: (1) the recommendation frequency of each soundscape, (2) the visiting condition of the soundscape and (3) the suitability for a group of people to listen at the same time, the evaluation soundscapes were selected proportionally in each category. 

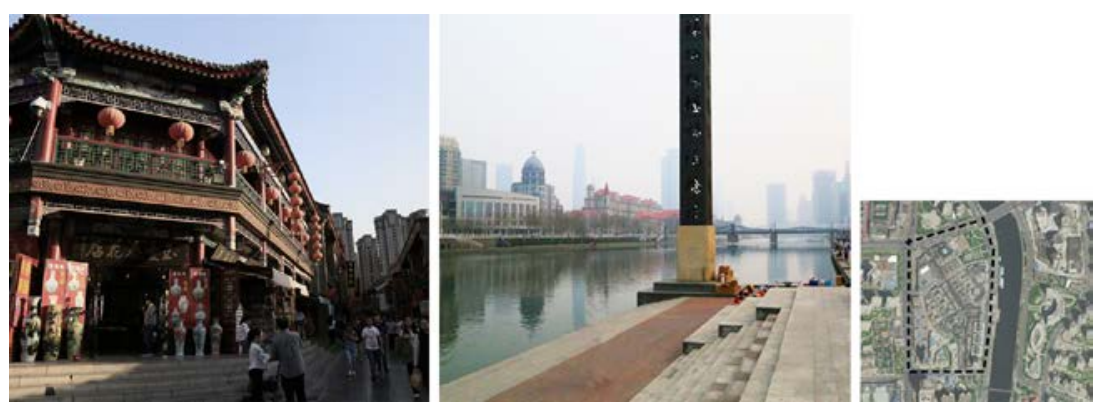

(a) Traditional Cultural Street This is a historic block with Chinese characteristics, which still conserves the traditional Chinese urban structure and organization layout.
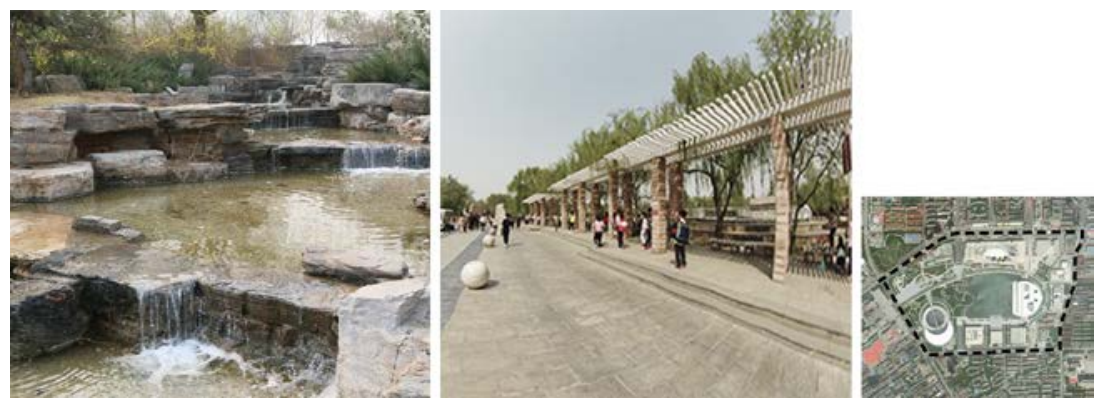

\section{(b) Civic Square}

This square is a large green space for leisure activities, surrounded by low buildings, and many children play in the square every day.
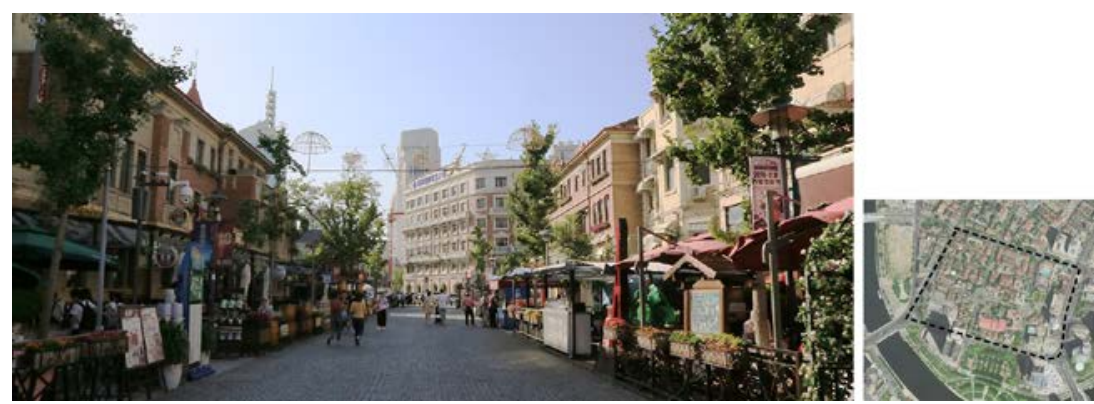

(c) Italian Style Street

This is the only street in Asia with Italian style. There are more than 200 European buildings in this area, which have been preserved for more than 100 years.
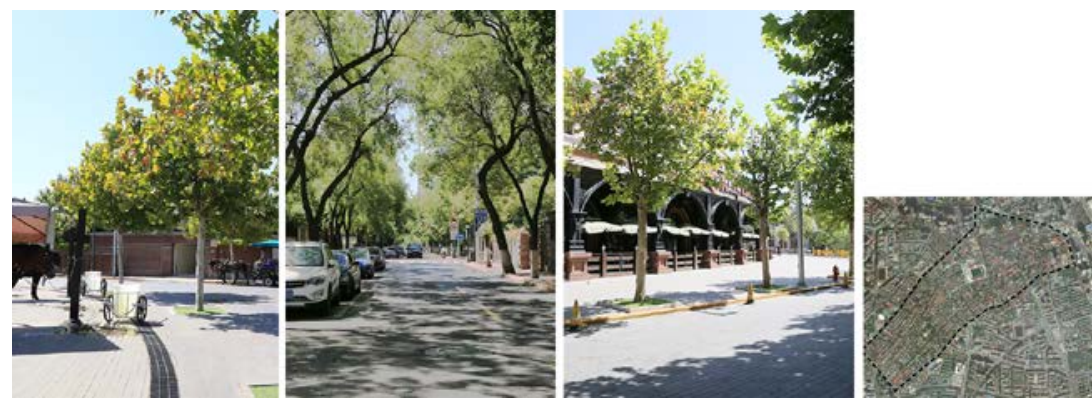

\section{(d) Five Old Street}

This is a famous scenic spot, with profound cultural and historic character. The horse carriage is still the one of the transportation tools in this area and the sound environment is relatively quiet in this block. 

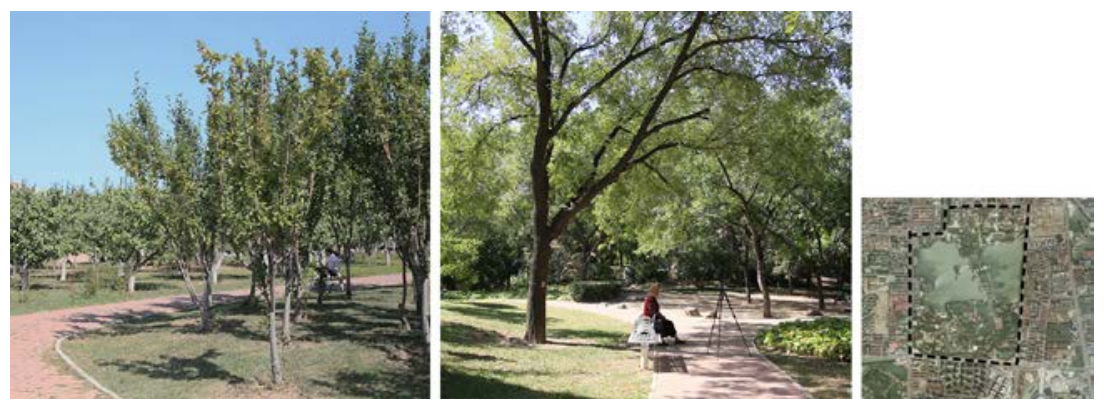

(e) Water Park

This is the largest park in Tianjin for citizens to relax. Its greening rate is high, and the air quality is good.
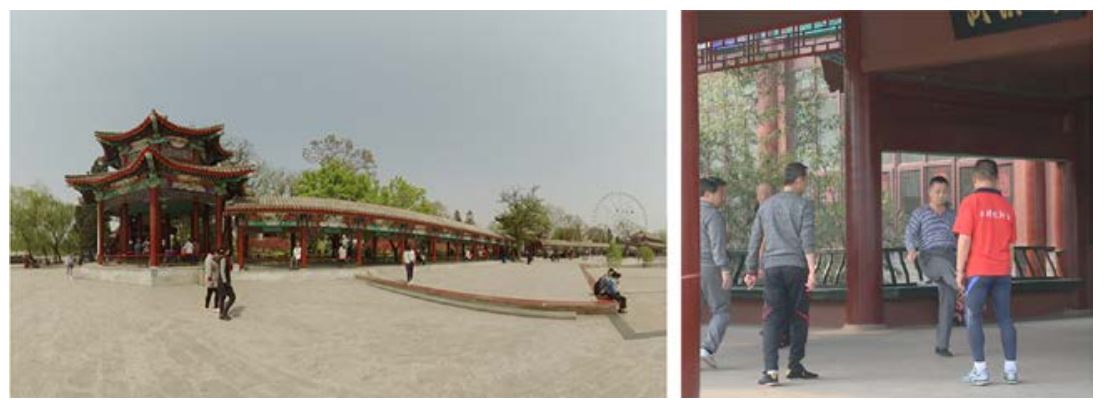

Fig. 1. Soundscapes to be evaluated during the group soundscape walk

During the soundscape walk in each location, the participants were asked to walk around for 30 min to experience the sound environment and then complete the questionnaire. There were three parts of the questionnaire: (1) the characteristics of soundscapes which would be evaluated using semantic differential method (Kang and Zhang, 2010), with seventeen pairs of adjectives as shown in Table 2. The adjective pairs were selected via the following steps. Firstly, thirty-two pairs of adjectives were selected according to previous soundscape studies (Gozalo et al., 2018) and our focus group interview on how people describe the urban soundscapes worthy of preservation. Then, through a preliminary test, fifteen pairs of adjectives were excluded since they were not easy to be understood or evaluated. (2) The identification of sound sources at each location. Participants were asked to evaluate whether they could hear the sound or not using a five-point verbal scale from not hear at all to completely dominant. Eight categories of sounds were listed in the questionnaire, including social/communal sounds (talking, bell, laughter, and sounds from human activities, etc.), 
animal sounds (bird songs and other sounds from non-domesticated animals), water sounds, plant sounds, music sounds (amplified music or instrument), sounds from natural phenomena (wind, rain, etc.), traditional folk sounds, and mechanical sounds (Brown et al., 2011b). (3) The visual context of the soundscape was evaluated from three aspects: visual comfort, visual openness and visual diversity (Hong and Jeon, 2014; Lindquist et al., 2016). A five-point bipolar rating scale was used.

Table 2 Semantic evaluation form used in the group soundscape walk

\begin{tabular}{ccccccc}
\hline & Very & Little & Neutral & Little & Very & \\
\hline Gentle & -2 & -1 & 0 & 1 & 2 & Harsh \\
Fast & -2 & -1 & 0 & 1 & 2 & Slow \\
Simple & -2 & -1 & 0 & 1 & 2 & Varied \\
Natural & -2 & -1 & 0 & 1 & 2 & Artificial \\
Strong & -2 & -1 & 0 & 1 & 2 & Weak \\
Far & -2 & -1 & 0 & 1 & 2 & Close \\
Pleasant & -2 & -1 & 0 & 1 & 2 & Unpleasant \\
Relaxed & -2 & -1 & 0 & 1 & 2 & Intensive \\
Harmonious & -2 & -1 & 0 & 1 & 2 & Disharmonious \\
Light & -2 & -1 & 0 & 1 & 2 & Heavy \\
Quiet & -2 & -1 & 0 & 1 & 2 & Noisy \\
Comfort & -2 & -1 & 0 & 1 & 2 & Discomfort \\
Impure & -2 & -1 & 0 & 1 & 2 & Pure \\
Like & -2 & -1 & 0 & 1 & 2 & Dislike \\
Vibrancy & -2 & -1 & 0 & 1 & 2 & Dull \\
Directional & -2 & -1 & 0 & 1 & 2 & Universal \\
Representative & -2 & -1 & 0 & 1 & 2 & Unrepresentative \\
\hline
\end{tabular}

\subsection{Data analysis}

All the data analysis was performed using SPSS software. Firstly, people's attitude towards soundscape preservation was analysed by calculating the average evaluation score and counting the frequency of each option, and the influence of demographic characteristics on the attitude was 
checked by the analysis of variance (ANOVA). Secondly, in order to identify the characteristics of the soundscapes worthy of preservation, principal components analysis (PCA) was employed. And finally, hierarchical cluster analysis (HCA) based on the characteristics of the soundscape worthy of preservation was conducted to describe the classification of them for further comparison.

\section{Results}

\subsection{Attitude towards soundscape preservation}

The average scores related to people's attitude towards the soundscape preservation are shown in Fig. 2. In addition, the results of ANOVA, indicating the influence of gender, age, occupation and education level on people's attitude, are shown in Table 3 . Regarding the attitude to the importance of soundscapes in improving the urban environment, $83 \%$ of the respondents realized that urban soundscape was important or very important in improving the overall environmental quality and female scored significantly higher on average than male. Regarding the attitude to soundscape deterioration, $90 \%$ of the respondents said they would feel sorry or very sorry if the soundscapes were damaged. The average attitude score of female was 4.4 , which was significantly higher than that of male. There was a significant difference among occupations, and the pensioners gave the highest scores of attitude to soundscape deterioration. This result indicated that the female and the pensioner showed great anxiety to the urban soundscape deterioration. Regarding the urgency of soundscape preservation, $79 \%$ of the respondents thought it was urgent or very urgent,

particularly respondents over 60 years old gave the highest score of 4.52, which showed the elderly 
were more worried about the urban soundscapes. However, respondents with higher educational level attached a significantly lower urgency of soundscape preservation. It was also interesting that, compared with other occupations, the professional urban planner and landscape architect gave the lowest evaluation on the preservation urgency.

Table 3 The difference on the attitude among the respondents with different occupation, education level, age, and gender.

\begin{tabular}{cccc}
\hline Sig.(2-tailed) & $\begin{array}{c}\text { Importance of soundscapes } \\
\text { in improving the } \\
\text { environment }\end{array}$ & $\begin{array}{c}\text { Attitude towards } \\
\text { soundscape deterioration }\end{array}$ & $\begin{array}{c}\text { Urgency evaluation of } \\
\text { soundscape preservation }\end{array}$ \\
\hline Gender & $.006^{* *}$ & $.002^{* *}$ & .233 \\
Occupation & .172 & $.002^{* *}$ & $.000^{* *}$ \\
Age groups & $.002^{*}$ & .248 & $.000^{* *}$ \\
Education level & .496 & .904 & $.004^{* *}$ \\
\hline
\end{tabular}

Where $\mathrm{p}<0.01$ was marked with ${ }^{* *}$ and $\mathrm{p}<0.05$ was marked with $*$

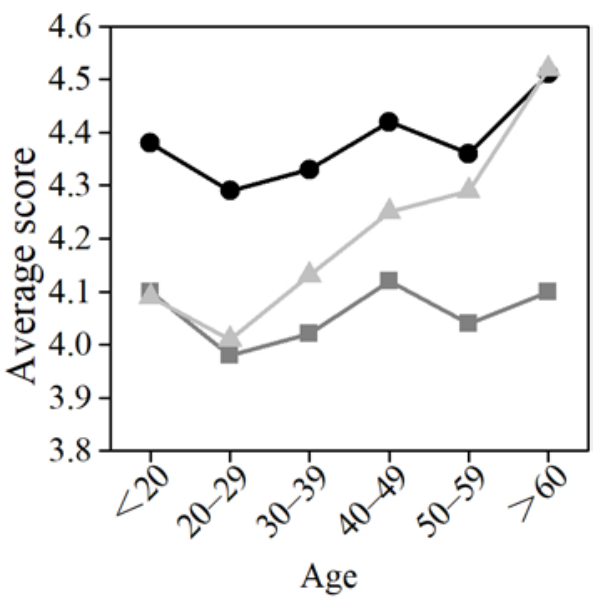

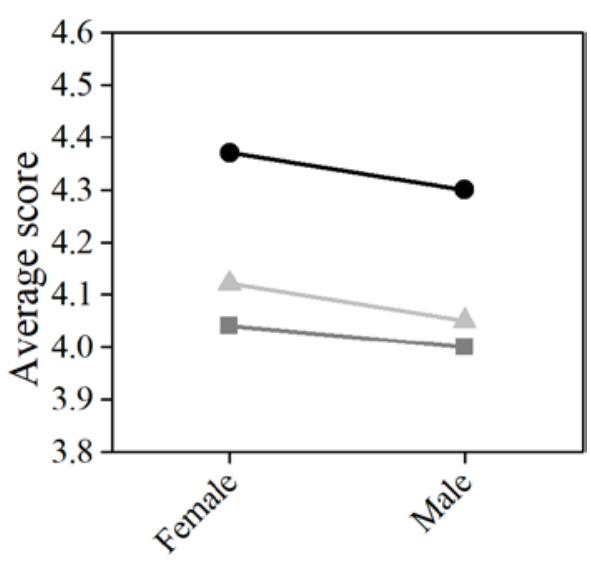

Gender 

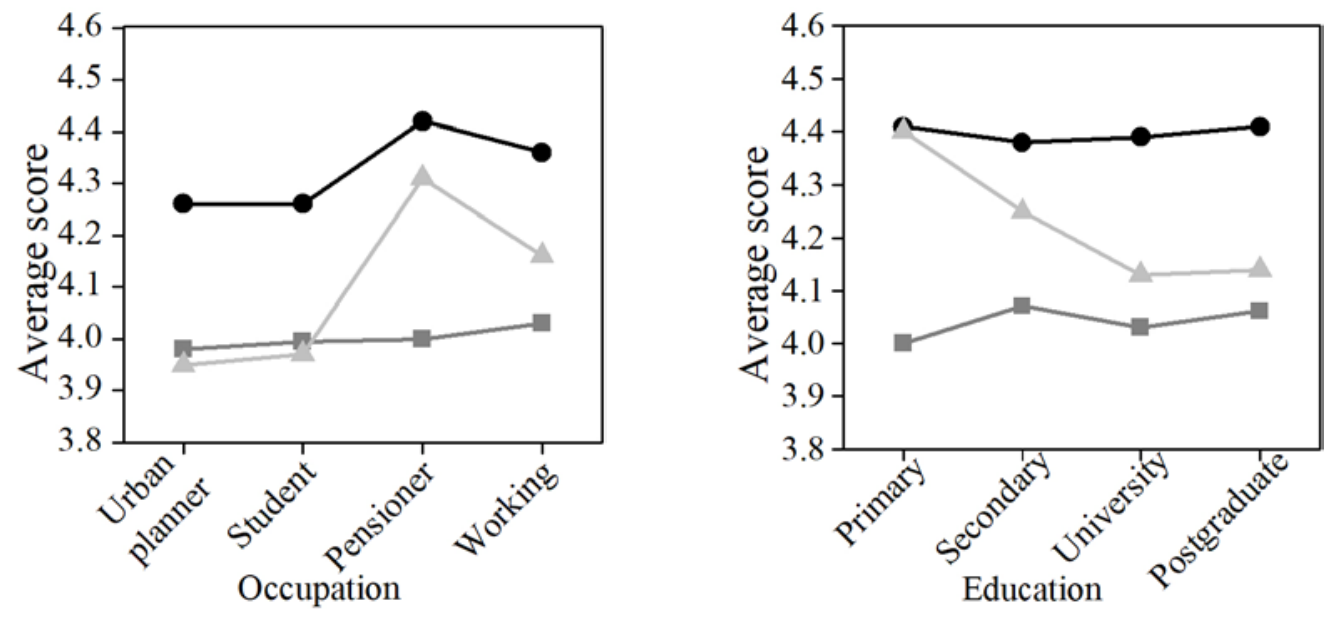

Fig. 2. Influence of respondents' demographic characteristics on their attitude towards soundscape preservation.

The values represent average scores on the attitude. The larger the score value is, the more positive subjective evaluation is shown.

Represents the respondents' cognition on the importance of soundscapes to improving the urban environment;

$\longrightarrow$ Represents the respondents' attitude towards the deterioration of urban soundscape;

Represents the respondents' attitude towards the urgency of soundscape preservation.

\subsection{Soundscapes worthy of preservation}

A total of 158 soundscapes were considered worthy of preservation according to the recommendations at the first stage. Among them, twenty-seven soundscapes were recommended more than twenty times (approximately 1\% of total respondents), as shown in Fig. 3. 


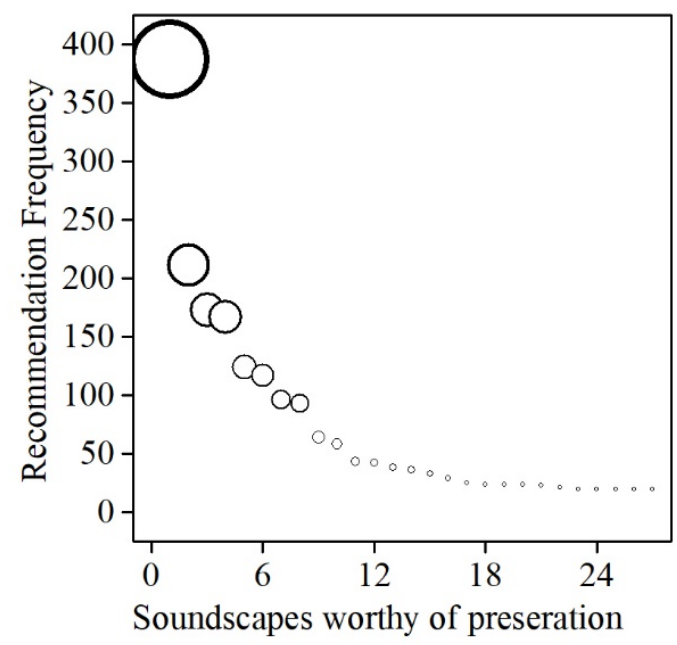

Fig. 3. Soundscapes worthy of preservation

(1. Water sound of Haihe River, 2. Birdsong in Water Park, 3. Bell of Tianjin Railway Station, 4. Music in Italian Style Street, 5. Music fountain in Civic Square, 6. Water sound in Civic Square, 7. Pedlary in Traditional Cultural Street, 8. Leaves rustling in alley, 9. Conversations in bystreet using a dialect, 10. Tranquil environment at Five Old Street, 11. Peking opera performance in Water Park, 12. Whistle on Haihe River, 13. Campus Broadcast, 14. Cicada sound in the campus 15. Sound of children playing in Civic Square, 16. Sound in Dabei temple, 17. Sound from fallen leaves due to wind blowing, 18. Carriage sound in Five Old Street, 19. Dance music in Water Park, 20. Crowd sound in Commercial Street, 21. Musical instrument sound in Water Park, 22. Running sound in Minyuan Stadium, 23. Choir in Xikai Church, 24. Reading sound in classroom, 25. Sound of skating on the surface of Haihe River, 26. Sound of the silversmith's old craft, and 27. Music in Jingyuan Garden)

The water sound of Haihe River was the most recommended soundscape. Haihe River is an important recreational landscape in Tianjin. Furthermore, as the mother river of Tianjin, it is associated with memories of the local people. Unfortunately, the water sound of the Haihe River is being masked by the increasing noise. Thus, respondents cherished it and expressed their desire to preserve this soundscape. The birdsong in the Water Park was also a soundscape that needed to be preserved, probably because it had many benefits such as being helpful for forming a good ecological environment (Machado et al., 2017). Some respondents who recommended the birdsong explained that the birdsong in the parks was a comfort soundscape, but it was rarely heard in the 
noisy cities. The bell of the Tianjin railway station was another important soundscape worthy of preservation probably because bell sound is usually regarded as a representative sound of a city (Schafer, 1980). This bell is more important than other bells because it lies in the railway station of Tianjin, the landmark of the city.

The top three sound source categories were social/communal sounds, animal sounds and water sounds, with the respective proportions of 34\%, 18\%, and $15 \%$. Similarly, these three sound source categories were also the top three in the Japanese survey, respectively accounting for 27\%, 23\%, and 19\%, of the total sound source categories (Ge and Hokao, 2004).

As for the distribution of the recommended soundscapes, most of them were located in urban parks, historic districts, and commercial areas, with the respective proportions of $66 \%, 20 \%$ and $14 \%$. People tended to suggest to preserve the soundscapes in urban parks, and one of the reasons might be that urban parks, as an important landscape to restore physical and mental health of citizens (Brown et al., 2014), usually contained comfortable soundscapes. Furthermore, some culture-related soundscapes located in the historic districts were considered worthy of preservation, possibly because they contained historic information and were regarded as cultural heritages of the city (Huang and Kang, 2015; Yelmi, 2016).

\subsection{Characteristics of soundscapes worthy of preservation}

The characteristics of soundscapes worthy of preservation were evaluated during the group soundscape walk. Based on the evaluations, five factors which could describe the characteristics 
of the soundscapes worthy of preservation were extracted, and they covered $68 \%$ of the total variance, as shown in Table 4. Factor 1 (32\%) was mainly associated with relaxation, including gentle-harsh, harmonious-disharmonious, comfort-discomfort, like-dislike, fast-slow, relaxedintensive, quiet-noisy and impure-pure. Factor 2 (15\%) was generally associated with vibrancy, including pleasant-unpleasant, light-heavy and vibrancy-dull. Factor 3 (8\%) was mostly associated with representativeness, including natural-artificial and representativeunrepresentative. Factor 4 (7\%) was principally related to strength, including far-close and strongweak. Factor 5 (6\%) was associated with richness, including simple-varied and directionaluniversal.

Table 4 Principal factor loadings of the PCA on perceived characteristics of soundscapes

\begin{tabular}{llllll}
\hline Indices & $1(32 \%)$ & $2(15 \%)$ & $3(8 \%)$ & $4(7 \%)$ & $5(6 \%)$ \\
\hline Gentle-Harsh & $\mathbf{0 . 6 1}$ & 0.21 & 0.21 & 0.34 & -0.29 \\
Fast-Slow & $\mathbf{- 0 . 6 4}$ & 0.42 & 0.13 & -0.33 & 0.07 \\
Relaxed-Intensive & $\mathbf{- 0 . 5 5}$ & -0.44 & -0.12 & -0.18 & 0.21 \\
Harmonious-Disharmonious & $\mathbf{0 . 5 6}$ & 0.52 & -0.01 & 0.22 & -0.11 \\
Quiet-Noisy & $\mathbf{- 0 . 7 1}$ & -0.12 & -0.30 & -0.20 & -0.05 \\
Comfort-Discomfort & $\mathbf{0 . 6 8}$ & 0.49 & 0.08 & -0.06 & 0.10 \\
Impure-Pure & $\mathbf{- 0 . 6 3}$ & -0.27 & -0.33 & 0.06 & -0.28 \\
Like-Dislike & $\mathbf{0 . 6 4}$ & 0.55 & 0.06 & 0.07 & -0.08 \\
Pleasant-Unpleasant & 0.13 & $\mathbf{0 . 7 3}$ & -0.02 & 0.10 & -0.07 \\
Light-Heavy & 0.24 & $\mathbf{0 . 6 9}$ & 0.16 & 0.13 & -0.23 \\
Vibrancy-Dull & 0.05 & $\mathbf{- 0 . 8 0}$ & 0.27 & 0.13 & 0.13 \\
Natural-Artificial & 0.23 & 0.09 & $\mathbf{0 . 7 2}$ & -0.20 & 0.24 \\
Representative-Unrepresentative & 0.15 & 0.15 & $\mathbf{- 0 . 7 6}$ & -0.11 & -0.16 \\
Strong-Weak & -0.39 & 0.12 & -0.26 & $\mathbf{- 0 . 6 3}$ & 0.07 \\
Far-Close & -0.23 & 0.10 & 0.08 & $\mathbf{0 . 8 9}$ & 0.07 \\
Simple-Varied & 0.07 & -0.17 & 0.39 & -0.14 & $\mathbf{0 . 6 9}$ \\
Directional-Universal & 0.02 & -0.01 & -0.20 & 0.17 & $\mathbf{0 . 8 5}$ \\
\hline
\end{tabular}

*Kaiser-Meyer-Olkin measure of sampling adequacy: 0.84, N: 276. These bold are called principal factor loading, and they represent the correlation coefficients of principal components and indices. 


\subsection{Classification of soundscapes worthy of preservation}

To improve the efficiency of the preservation work, soundscapes with similar characteristics were classified into one cluster. Many algorithms have been used by previous researchers to classify soundscapes (Morgan et al., 2019). Among them, the HCA method yielded relatively stable results without requiring huge amounts of data (Jeon and Hong, 2015). As shown in Fig. 4, the soundscapes worthy of preservation are classified into five clusters based on the HCA method. The typical soundscapes of cluster A were sounds of leaves rustling in alley, water sound of Haihe River and tranquil environment at Five Old Street. The soundscapes belonging to cluster B included birdsong in Water Park and water sound in Civic Square. Cluster C mainly included sounds of children playing in Civic Square and dance music in Water Park. Soundscapes of cluster D were carriage sound in Five Old Street, musical instrument sound in Water Park, music in Italian Style Street and Peking opera performance in Water Park. The sound of pedlary in Traditional Culture Street was the typical soundscape of cluster E. Since the HCA method was used, the five soundscape clusters were not compatible and overlapped. 


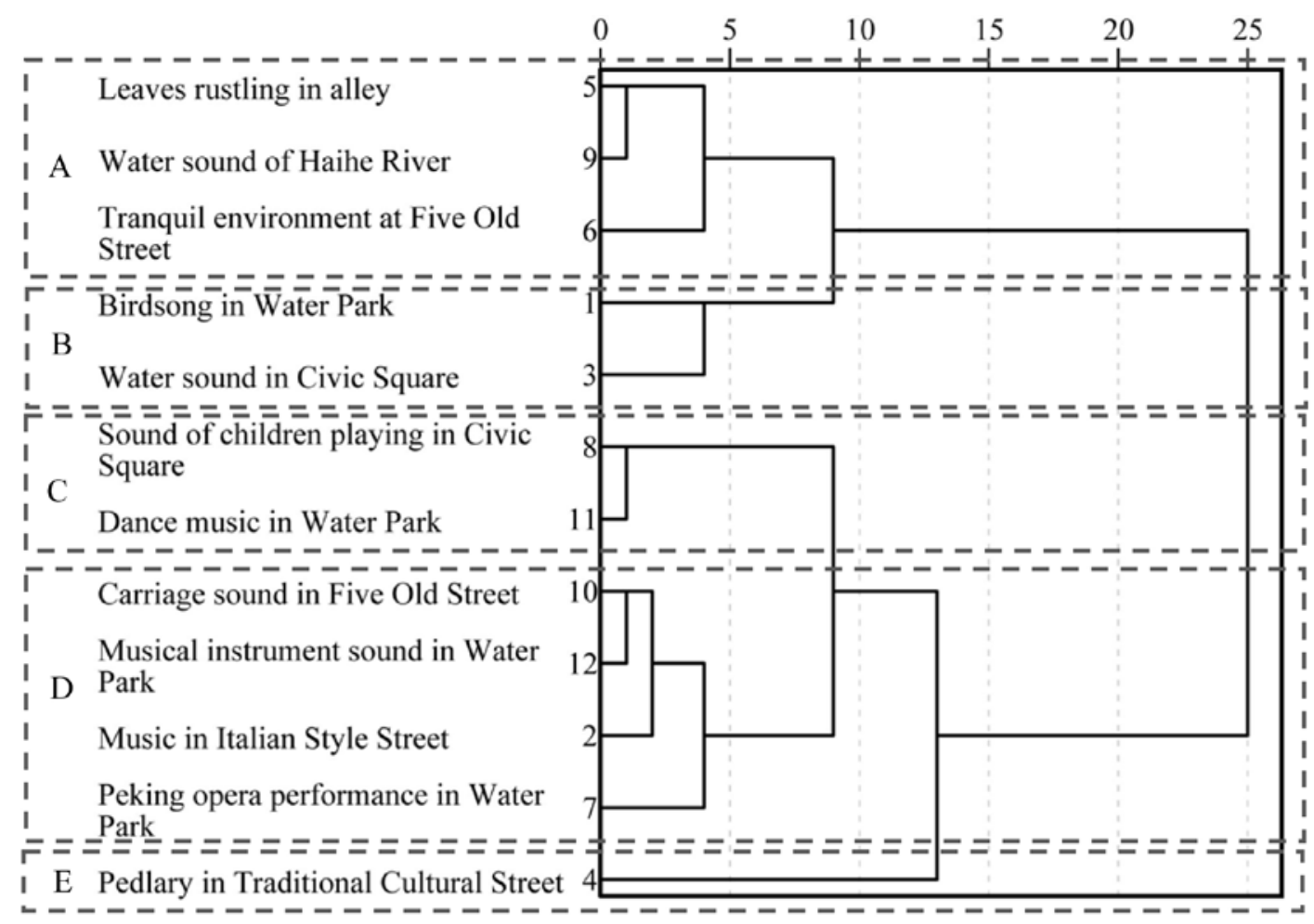

Fig. 4. Classification results of soundscapes worthy of preservation

The characteristics of the five soundscape clusters are shown in Fig. 5. The average scores of cluster A were 0.55 (relaxation), - -0.40 (vibrancy), -0.60 (representativeness), -0.46 (strength) and 0.20 (richness). This cluster was natural soundscape, and its dominant characteristic was relaxation. Thus, this cluster could be named as relaxing nature soundscape. The average scores of cluster B were 0.36 (relaxation), 0.81 (vibrancy), -0.74 (representativeness), -0.27 (strength), and 0.36 (richness). This cluster was also natural soundscape, and its dominant characteristic was vibrancy. This type of soundscape could be called vibrant nature soundscape. It would appear that reducing the vibrancy and strength may lead to soundscapes that can provide better relaxation. The assessment scores of cluster C were -0.63 (relaxation), 0.59 (vibrancy), 0.19 (representativeness), -0.42 (strength), and -0.45 (richness). This cluster mainly represented the sound of recreational 
activities. Compared with other artificial soundscapes, they are unrepresentative, but they are the necessary sounds of a city. This cluster was named as vibrant human activity soundscape. The evaluation scores of cluster D were 0.12 (relaxation), -0.35 (vibrancy), 0.46 (representativeness), -0.01 (strength), and -0.24 (richness). The sound mark of this cluster was the culture-related sound. Thus, it was named as culture-related soundscape. Meanwhile, based on the higher score of the representativeness characteristic, this cluster was regarded as one of representative soundscapes of the city. The characteristics of cluster $\mathrm{E}$ were -1.31 (relaxation), 0.15 (vibrancy), 0.92 (representativeness), 0.63 (strength) and 1.07 (richness). It could be referred as traditional soundscape, and its representativeness characteristic was outstanding. This cluster had lower sense of relaxation and higher strength, but it was still one of the soundscapes worthy of preservation. This was possibly because participants not only considered acoustic comfort, but also the value of traditional or cultural soundscapes when deciding whether to preserve the soundscape or not.

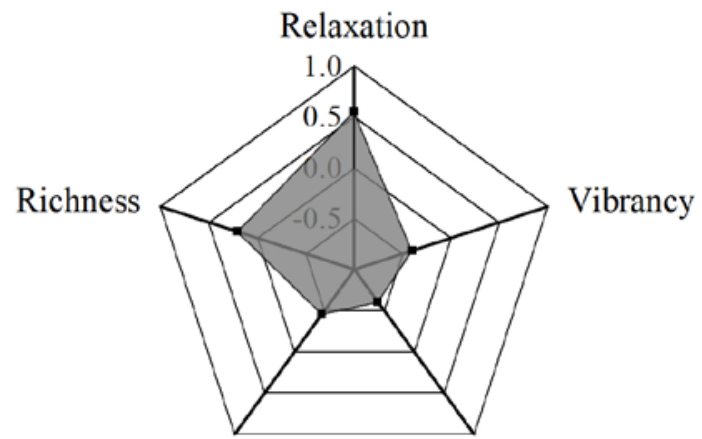

Strength
Representativeness

$\longrightarrow$ Relaxing nature soundscape

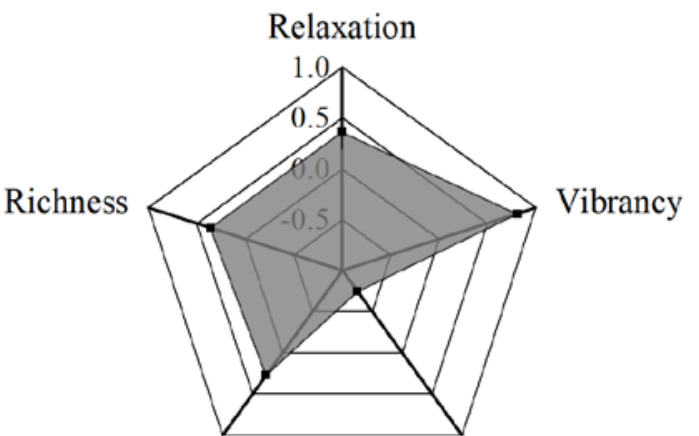

Strength

- Vibrant nature soundscape 


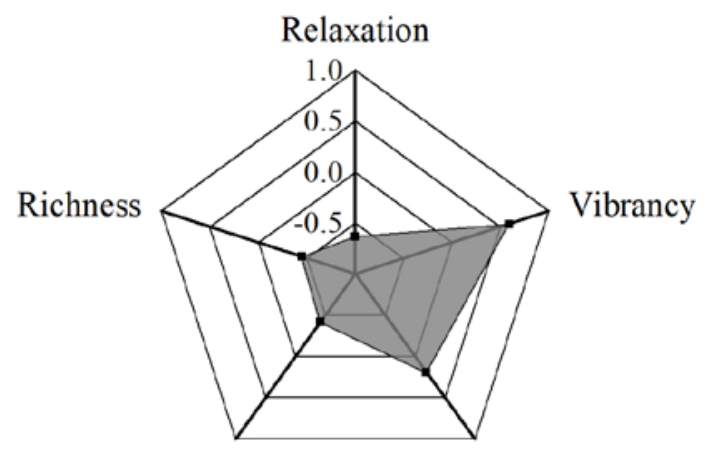

Strength

Representativeness

$\longrightarrow$ Vibrant human activity soundscape

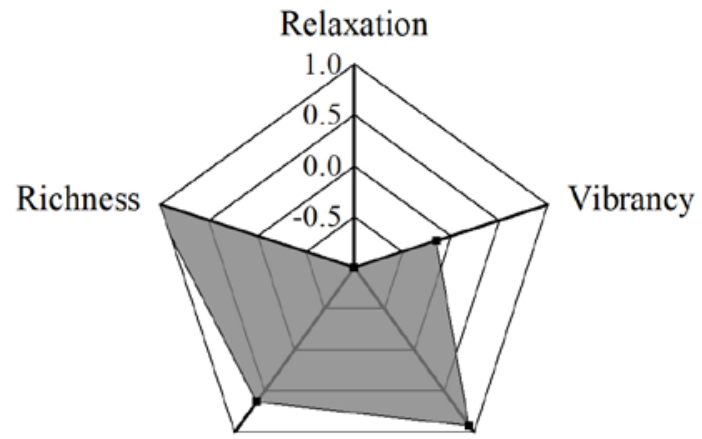

Strength Representativeness

$\longrightarrow$ Traditional soundscape

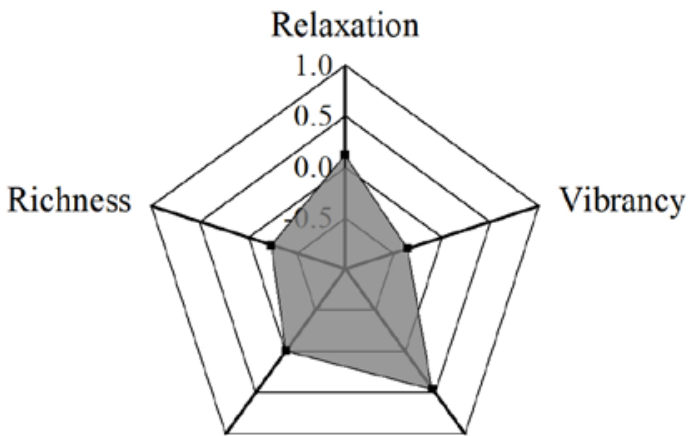

Strength

$\longrightarrow$ Culture-related soundscape

Fig. 5. Characteristics of the five soundscape clusters

Sound source is an important influencing factor on the characteristic of soundscape (Hong and Jeon, 2017). These soundscapes worthy of preservation could unite various sound sources in a harmonious manner. Traffic noise is the most prominent background sound of modern cities, and appeared in the above five soundscape clusters. It even constituted the dominant sound of the relaxing nature soundscape. Nevertheless, relaxation was still the dominant characteristic of this soundscape cluster. It seems that although the sound mark was not the dominant sound of the soundscape, it could still determine the characteristic of the soundscape. However, this finding did not agree with some previous research findings (Pérez-Martínez et al., 2018), which showed that 
the dominant sound was the most important element affecting soundscape quality. A possible explanation for this result may be that people are more concerned about the information transmitted by soundscape rather than the soundscape quality or acoustic comfort when evaluating soundscapes worthy of preservation.

Moreover, visual comfort, visual diversity and visual openness of these soundscapes worthy of preservation were evaluated. Fig. 6 shows that the average evaluation scores of the three indicators are $0.71,0.20$ and 0.69 respectively. According to the result of ANOVA, the evaluation scores of visual comfort and openness were significantly higher $(\operatorname{sig}<0.01)$ than that of the visual diversity. This result indicated that the soundscapes worthy of preservation usually had a comfortable and open visual scene instead of a diverse visual scene.

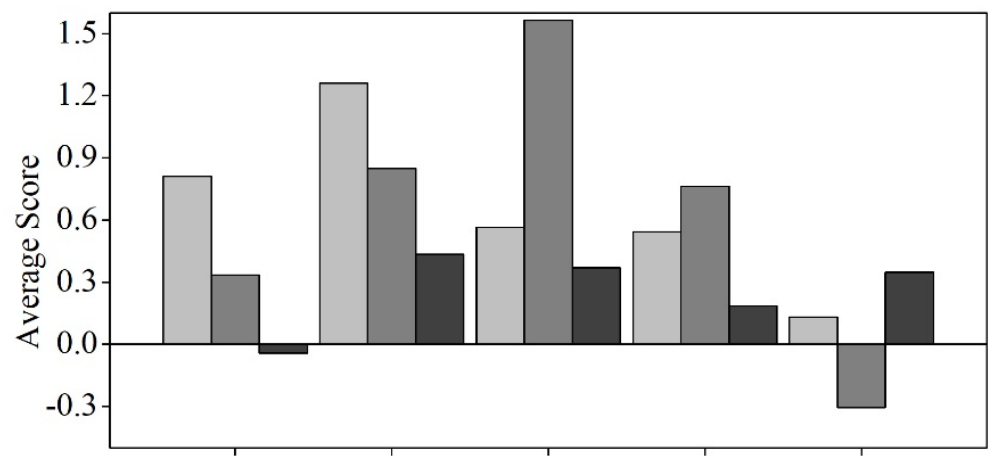

Cluster A Cluster B Cluster C Cluster D Cluster E Soundscape Cluster

Visual comfort $\square$ Visual openness $\square$ Visual diversity

Fig. 6. Average evaluation scores of visual scenes of soundscapes worthy of preservation 


\section{Discussion}

\subsection{Analysis of the characteristics of soundscapes worthy of preservation}

In this study, five principal factors were extracted to describe the characteristics of the soundscapes worthy of preservation, and a total of $68 \%$ of the variance was explained. When the total variance was basically equal or even more, the number of principle factors in many previous studies were generally 2-4, as shown in Table 5 . This indicated that the characteristics of the soundscapes worthy of preservation were more complex. Meanwhile, the weight of the relaxation characteristic was higher than that of other factors, which reflected the urgent need for sound environment with a high sense of relaxation in modern society. The reason may be that Chinese urban residents (including children) are generally feeling pressured and stressed (Shu and Ma, 2018). Thus, preservation of a good sound environment that promotes recovery and relaxation is of great importance.

Table 5 The principal perceived characteristics of urban soundscape extracted in some typical studies

\begin{tabular}{|c|c|c|c|c|}
\hline Author & Study area & Factors & Method & Reference \\
\hline \multirow{4}{*}{ Brambilla } & \multirow{4}{*}{ Urban squares } & Enclosed (72\%) & \multirow{4}{*}{ Laboratory study } & \multirow{4}{*}{ (Brambilla et al., 2013) } \\
\hline & & Calm (20\%) & & \\
\hline & & Spatiality (27\%) & & \\
\hline & & Environment (17\%) & & \\
\hline \multirow{2}{*}{ Rebecca } & \multirow{2}{*}{ Urban } & Calmness (60\%) & \multirow{2}{*}{ Laboratory study } & \multirow{2}{*}{ (Cain et al., 2013) } \\
\hline & & Vibrancy (20\%) & & \\
\hline \multirow{3}{*}{ Kawai } & \multirow{3}{*}{ Urban } & Preference (25\%) & \multirow{3}{*}{ Laboratory study } & \multirow{3}{*}{ (Kawai et al., 2004) } \\
\hline & & Activity (17\%) & & \\
\hline & & Daily life (9\%) & & \\
\hline
\end{tabular}




\begin{tabular}{lllll}
\hline \multirow{4}{*}{ Kang } & Urban open public & Relaxation (26\%) & & \\
& Space & $\begin{array}{l}\text { Communication (12\%) } \\
\text { Spatiality (8\%) } \\
\text { Dynamics (7\%) }\end{array}$ & Social survey & (Kang and Zhang, 2010) \\
& & Pleasantness (50\%) & Laboratory & (Axelsson et al., 2010) \\
Axelsson & Urban & Eventfulness (18\%) & study & \\
& & & &
\end{tabular}

\subsection{Suggestions on soundscape preservation work}

The key aim of urban planning is to create, reproduce, or shape an environment with regional characteristics. All senses should be dealt with to the same degree, thus the auditory perception should also be taken into account (Hague and Jenkins, 2004). Most respondents acknowledged the importance of the preservation of soundscape, but the process ultimately depends on policy makers. Accordingly, the process identified in this study can provide a reference for other regions to carry out soundscape preservation investigation. The first step is the identification of the target soundscapes for preservation work. There are various types of soundscapes to be preserved, and they are significantly different in acoustic characteristics or cultural and social connotations. Secondly, to propose targeted preservation measures and improve the efficiency of the preservation work, the characteristics and classifications of these soundscapes also need to be analysed. Finally, it is also necessary to monitor and evaluate the soundscapes worthy of preservation continually.

The measures of preservation work depended on the soundscape characteristics (Brown and Muhar, 2004). The following aspects should be considered: (1) sound mark plays a decisive role in the evaluation of soundscapes that are deemed worthy of preservation. Therefore, the key principle of soundscape preservation is to preserve the sound marks and their propagation spaces. 
(2) According to the soundscape characteristic, the natural soundscapes ought to be classified into two clusters. One cluster has the dominant characteristic of relaxation. This type has a lower strength and is easily affected by noise. To preserve such soundscapes, the surrounding environment needs to be rationally planned. Dynamic and tranquil spaces should be separated, and ambient noise should be controlled. To control those unwanted sound, many effective measures have been put forward by previous studies (Nilsson et al., 2013), such as using barriers, trees, shrubs and bushes. The other cluster have the dominant characteristic of vibrancy, with dynamic natural sounds such as birdsong or running water. To preserve this type of soundscape, more attention should be placed on the ecological environment. Increasing the number of plants to attract more birds or insects is helpful. These above suggestions are a supplement to the effective methods put forward by previous studies (Cerwén et al., 2017). (3) For soundscapes whose dominant characteristic are the representativeness, their sound marks are sounds with cultural meanings or local characteristics. However, with the urbanisation, such soundscapes are often overlooked. In order to conserve traditional activities or customs that produce such sounds, the participation of residents is very important. The "100 Soundscapes of Japan: Preserving Our Heritage" was a successful project which have far-reaching significance to arouse the public's interest to discover the value of soundscapes. Governments could conduct some similar projects to increase publicity to make people aware of the significance of such soundscapes. Since those clusters of soundscape are mostly distributed in historic areas, when the historic districts are reconstructed, full consideration should be given to the impact on the soundscapes. This ultimately refers to a conservation of the historic areas at a deeper level. 
Despite these findings, there were limitations to this research. One of them is that soundscapes worthy of preservation may vary in different countries, cities and regions owing to the socialcultural diversity, thus this work need to perform cross-cultural comparisons on a global scale. Moreover, since environmental factors such as temperature and humidity have effects on the soundscape evaluation, the soundscape walk may be carried out in different seasons involving different group of participants in further studies to get more detailed knowledge of people's cognition on soundscape preservation.

\section{Conclusions}

This study investigated the characteristics of urban soundscapes worthy of preservation. The main conclusions can be summarised as follows:

(1) In total, $79 \%$ of the respondents thought the soundscape preservation was urgent or very urgent, with an average score of 4.04 points (five-point numeric scale was used and five meant very urgent). As for the attitude to the soundscape preservation, statistically significant difference was shown among the respondents with different demographic characteristics. In terms of age, respondents aged 60 or older had the most supportive attitude towards soundscape preservation work. Compared with people in other professions, pensioners were more eager to support preservation efforts. With improvements in educational level, people tended to assign lower evaluation scores to the urgency of soundscape preservation. Additionally, female were more enthusiastic about the urban soundscape preservation. 
(2) The urban soundscapes worthy of preservation recommended by the respondents were situated in various locations. Among them, parks and historic districts were the most important urban contexts for these soundscapes. Furthermore, the top three sound source categories of these recommended soundscapes were social/communal sounds (talking, bells, laughter, and sounds from human activities), animal sounds (birdsongs, and other sounds from non-domesticated animals), and water sounds.

(3) Five principle factors were identified to describe the characteristics of the soundscapes worthy of preservation, including relaxation, vibrancy, representativeness, strength, and richness. These five factors covered $68 \%$ of the total variance, while half of the variance was explained by the relaxation factor.

(4) Based on the characteristics of the soundscapes worthy of preservation, these soundscapes were classified into five clusters. They were clusters of relaxing nature soundscape, vibrant nature soundscape, vibrant human activity soundscape, culture-related soundscape, and traditional soundscape. The characteristics of the soundscapes worthy of preservation were dependent on their sound marks rather than the dominant sounds. For the evaluation of the visual scenes, these soundscapes usually had comfort and open visual scenes instead of diverse visual scenes.

Overall, these results are expected to help urban planners and relevant government sectors to design and preserve urban soundscapes. It is evident that the preservation of sound marks is a key principle of soundscape preservation work. Correspondingly, the preservation measures should depend on their characteristics. Urban soundscape preservation is not only an important aspect of urban landscape preservation, but is also an essential reference for urban landscape design. 


\section{Funding}

This work was supported by the National Natural Science Foundation of China [grant numbers. 51678401 and 51978454].

\section{Acknowledgments}

We would like to express our appreciation for all participants who participated in the social survey and the soundscape walk.

\section{References}

Alazaizeh, M.M., Hallo, J.C., Backman, S.J., Norman, W.C., Vogel, M.A., 2019. Giving voice to heritage tourists: indicators of quality for a sustainable heritage experience at Petra, Jordan. J. Tour. Cult. Chang. 17, $269-284$. https://doi.org/10.1080/14766825.2018.1455693.

Aletta, F., Oberman, T., Kang, J., 2018. Associations between positive health-related effects and soundscapes perceptual constructs: A systematic review. Int. J. Environ. Res. Public Health 15, 2392.

https://doi.org/10.3390/ijerph15112392.

Axelsson, Ö., Nilsson, M.E., Berglund, B., 2010. A principal components model of soundscape perception. J. Acoust. Soc. Am. 128, 2836.

Brambilla, G., Maffei, L., Di Gabriele, M., Gallo, V., 2013. Merging physical parameters and laboratory subjective ratings for the soundscape assessment of urban squares. J. Acoust. Soc. Am. 134, 782-790.

https://doi.org/10.1121/1.4768792.

Brown, A., Muhar, A., 2004. An approach to the acoustic design of outdoor space. J. Environ. Planning Manage. 47, 827-842. https://doi.org/doi.org/10.1080/0964056042000284857.

Brown, A.L., Kang, J., Gjestland, T., 2011a. Towards standardization in soundscape preference assessment. Appl. Acoust. 72, 387-392. https://doi.org/10.1016/j.apacoust.2011.01.001.

Brown, A.L., Kang, J.A., Gjestland, T., 2011b. Towards standardization in soundscape preference assessment. Appl. Acoust. 72, 387-392. https://doi.org/10.1016/j.apacoust.2011.01.001.

Brown, G., Schebella, M.F., Weber, D., 2014. Using participatory GIS to measure physical activity and urban park benefits. Landsc. Urban Plan. 121, 34-44. https://doi.org/10.1016/j.landurbplan.2013.09.006.

Cain, R., Jennings, P., Poxon, J., 2013. The development and application of the emotional dimensions of a soundscape. Appl. Acoust. 74, 232-239. https://doi.org/10.1016/j.apacoust.2011.11.006.

Cerwén, G., Kreutzfeldt, J., Wingren, C., 2017. Soundscape actions: A tool for noise treatment based on three 
workshops in landscape architecture. Front. Archit. Res. 6, 504-518.

https://doi.org/doi.org/10.1016/j.foar.2017.10.002.

de la Prida, D., Pedrero, A., Navacerrada, M.A., Diaz, C., 2019. Relationship between the geometric profile of the city and the subjective perception of urban soundscapes. Appl. Acoust. 149, 74-84.

https://doi.org/10.1016/j.apacoust.2019.01.025.

Dumyahn, S.L., Pijanowski, B.C., 2011. Soundscape conservation. Landsc. Ecol. 26, 1327-1344.

https://doi.org/10.1007/s10980-011-9635-x.

Francis, C.D., Newman, P., Taff, B.D., White, C., Monz, C.A., Levenhagen, M., Petrelli, A.R., Abbott, L.C., Newton, J., Burson, S., Cooper, C.B., Fristrup, K.M., McClure, C.J.W., Mennitt, D., Giamellaro, M., Barber, J.R., 2017. Acoustic environments matter: Synergistic benefits to humans and ecological communities. J. Environ. Manage. 203, 245-254. https://doi.org/10.1016/j.jenvman.2017.07.041.

Furumo, P.R., Aide, T.M., 2019. Using soundscapes to assess biodiversity in Neotropical oil palm landscapes. Landsc. Ecol. 34, 911-923. https://doi.org/10.1007/s10980-019-00815-w.

Ge, J., Hokao, K., 2004. Research on the sound environment of urban open space from the viewpoint of soundscape-A case study of Saga Forest Park, Japan. Acta Acust. United Acust. 90, 555-563.

https://doi.org/10.1134/1.1739504.

Gozalo, G.R., Morillas, J.M.B., González, D.M., Moraga, P.A., 2018. Relationships among satisfaction, noise perception, and use of urban green spaces. Sci. Total. Environ. 624, 438-450.

https://doi.org/10.1016/j.scitotenv.2017.12.148.

Hague, C., Jenkins, P., 2004. Place identity, participation and planning. Routledge, London.

Han, M.-H., Oh, Y.-K., 2008. The Significance and Direction on the Preservation of Sound Environment in KoreaFocused on the Comparison of 100 Soundscapes of Japan. Periodical, 43-50.

http://www.koreascience.or.kr/article/JAKO200830360545345.page

Han, X., Huang, X., Liang, H., Ma, S., Gong, J., 2018. Analysis of the relationships between environmental noise and urban morphology. Environ. Pollut. 233, 755-763. https://doi.org/doi.org/10.1016/j.envpol.2017.10.126.

Hiramatsu, K., 2006. A review of soundscape studies in Japan. Periodical, 857-864.

https://www.ingentaconnect.com/content/dav/aaua/2006/00000092/00000006/art00002

Hong, J.Y., Jeon, J.Y., 2014. The effects of audio-visual factors on perceptions of environmental noise barrier performance. Landsc. Urban Plan. 125, 28-37. https://doi.org/10.1016/j.landurbplan.2014.02.001.

Hong, J.Y., Jeon, J.Y., 2017. Exploring spatial relationships among soundscape variables in urban areas: A spatial statistical modelling approach. Landsc. Urban Plan. 157, 352-364.

https://doi.org/10.1016/j.landurbplan.2016.08.006.

Huang, L., Kang, J., 2015. The sound environment and soundscape preservation in historic city centres—the case study of Lhasa. Environ. Plann. B Plann. Des. 42, 652-674. https://doi.org/10.1068/b130073p.

Iglesias, C., Diaz, L., Soliño, M., 2014. Noise pollution in national parks: Soundscape and economic valuation. Landsc. Urban Plan. 123, 1-9. https://doi.org/10.1016/j.landurbplan.2013.11.006.

Irvine, K.N., Devine-Wright, P., Payne, S.R., Fuller, R.A., Painter, B., Gaston, K.J., 2009. Green space, soundscape and urban sustainability: an interdisciplinary, empirical study. Local Environ. 14, 155-172.

https://doi.org/10.1080/13549830802522061.

Järviluoma, H., 2000. Acoustic environments in change: five village soundscapes revisited. Periodical, 25.

https://researchers.mq.edu.au/en/publications/acoustic-environments-in-change-and-five-village-soundscapes 
Jeon, J.Y., Hong, J.Y., 2015. Classification of urban park soundscapes through perceptions of the acoustical environments. Landsc. Urban Plan. 141, 100-111. https://doi.org/10.1016/j.landurbplan.2015.05.005.

Jeon, J.Y., Hong, J.Y., Lee, P.J., 2013. Soundwalk approach to identify urban soundscapes individually. J. Acoust. Soc. Am. 134, 803-812. https://doi.org/10.1121/1.4807801.

Kang, J., 2006. Urban sound environment. CRC Press, London.

Kang, J., Zhang, M., 2010. Semantic differential analysis of the soundscape in urban open public spaces. Build.

Environ. 45, 150-157. https://doi.org/10.1016/j.buildenv.2009.05.014.

Kato, K., 2009. Soundscape, cultural landscape and connectivity. sites: new series 6, 80-102.

https://doi.org/10.11157/sites-vol6iss2id123.

Kawai, K., Kojima, T., Hirate, K., Yasuoka, M., 2004. Personal evaluation structure of environmental sounds: experiments of subjective evaluation using subjects' own terms. J. Sound. Vib. 277, 523-533.

https://doi.org/10.1016/j.jsv.2004.03.013.

Lindquist, M., Lange, E., Kang, J., 2016. From 3D landscape visualization to environmental simulation: The contribution of sound to the perception of virtual environments. Landsc. Urban Plan. 148, 216-231.

https://doi.org/10.1016/j.landurbplan.2015.12.017.

Liu, F., Kang, J., 2016. A grounded theory approach to the subjective understanding of urban soundscape in Sheffield. Cities 50, 28-39. https://doi.org/10.1016/j.cities.2015.08.002.

Liu, J., Wang, Y., Zimmer, C., Kang, J., Yu, T., 2019. Factors associated with soundscape experiences in urban green spaces: A case study in Rostock, Germany. Urban For. Urban Green. 37, 135-146.

https://doi.org/10.1016/j.ufug.2017.11.003.

Liu, J., Xiong, Y., Wang, Y., Luo, T., 2018a. Soundscape effects on visiting experience in city park: A case study in Fuzhou, China. Urban For. Urban Green. 31, 38-47. https://doi.org/10.1016/j.ufug.2018.01.022.

Liu, X., Gao, T., Wang, X., 2018b. Tianjin, Regional Innovation Index of China: 2017. Springer, pp. 163-166.

Machado, R.B., Aguiar, L., Jones, G., 2017. Do acoustic indices reflect the characteristics of bird communities in the savannas of Central Brazil? Landsc. Urban Plan. 162, 36-43. https://doi.org/10.1016/j.landurbplan.2017.01.014. Marinelli, M., 2010. Internal and external spaces: The emotional capital of Tianjin’s Italian concession. Emotion, Space and Society, 9. https://doi.org/10.1016/j.emospa.2010.01.009.

Miller, N.P., 2008. US National Parks and management of park soundscapes: A review. Appl. Acoust. 69, 77-92. https://doi.org/10.1016/j.apacoust.2007.04.008.

Morgan, M.M., Moriarty, V., Braasch, J., 2019. Automatic environmental soundscape classification of continuous field recordings around Lake George, NY. J. Acoust. Soc. Am. 145, 1737-1737. https://doi.org/10.1121/1.5101369. Nilsson, M., Klæboe, R., Bengtsson, J., Forssén, J., Hornikx, M., Van der Aa, B., Rådsten-Ekman, M., Defrance, J.r.m., Jean, P., Koussa, F., 2013. Novel Solutions for Quieter and Greener Cities. Periodical, http://publications.lib.chalmers.se/records/fulltext/208780/local_208780.pdf

Ou, D., Mak, C.M., Pan, S., 2017. A method for assessing soundscape in urban parks based on the service quality measurement models. Appl. Acoust. 127, 184-193. https://doi.org/10.1016/j.apacoust.2017.06.006.

Pérez-Martínez, G., Torija, A.J., Ruiz, D.P., 2018. Soundscape assessment of a monumental place: A methodology based on the perception of dominant sounds. Landsc. Urban Plan. 169, 12-21.

https://doi.org/10.1016/j.landurbplan.2017.07.022.

Pijanowski, B.C., Farina, A., 2011. Introduction to the special issue on soundscape ecology. Landsc. Ecol. 26, 12091211. https://doi.org/10.1007/s10980-011-9655-6. 
Puyana, R., Virginia, Maffei, L., Brambilla, G., Ciaburro, G., 2016. Modelling the soundscape quality of urban waterfronts by artificial neural networks. Appl. Acoust. 111, 121-128.

https://doi.org/10.1016/j.apacoust.2016.04.019.

Quintero, G., Balastegui, A., Romeo, J., 2018. Annual traffic noise levels estimation based on temporal stratification. J. Environ. Manage. 206, 1-9. https://doi.org/10.1016/j.jenvman.2017.10.008.

Rey, G., G., Barrigon, M., J. M., 2017. Perceptions and effects of the acoustic environment in quiet residential areas. J. Acoust. Soc. Am. 141, 2418. https://doi.org/10.1121/1.4979335.

Schafer, R.M., 1980. The tuning of the world: Toward a theory of soundscape design. University of Pennsylvania Press Philadelphia, PA, USA.

Schafer, R.M., 1993. The soundscape: Our sonic environment and the tuning of the world. Simon and Schuster. Shu, S., Ma, H., 2018. The restorative environmental sounds perceived by children. J. Environ. Psychol. 60, 72-80. https://doi.org/doi.org/10.1016/j.jenvp.2018.10.011.

Torigoe, K., 2003. Insights taken from three visited soundscapes in Japan, Conf. proceedings “Acoustic Ecology”, Australian Forum for Acoustic Ecology/World Forum for Acoustic Ecology. Citeseer, Melbourne, Australia.

Van Renterghem, T., 2019. Towards explaining the positive effect of vegetation on the perception of environmental noise. Urban For. Urban Green. 40, 133-144. https://doi.org/10.1016/j.ufug.2018.03.007.

Weinzimmer, D., Newman, P., Taff, D., Benfield, J., Lynch, E., Bell, P., 2014. Human Responses to Simulated Motorized Noise in National Parks. Leisure Sci. 36, 251-267. https://doi.org/10.1080/01490400.2014.888022.

Yelmi, P., 2016. Protecting contemporary cultural soundscapes as intangible cultural heritage: sounds of Istanbul. Int. J. Herit. Stud. 22, 302-311. https://doi.org/10.1080/13527258.2016.1138237.

Yu, B.Y., Kang, J., Ma, H., 2016. Development of Indicators for the Soundscape in Urban Shopping Streets. Acta Acust. United Acust. 102, 462-473. https://doi.org/10.3813/aaa.918965.

Zhang, X., Ba, M., Kang, J., Meng, Q., 2018. Effect of soundscape dimensions on acoustic comfort in urban open public spaces. Appl. Acoust. 133, 73-81. https://doi.org/10.1016/j.apacoust.2017.11.024.

Zhao, J., Xu, W., Ye, L., 2018. Effects of auditory-visual combinations on perceived restorative potential of urban green space. Appl. Acoust. 141, 169-177. https://doi.org/10.1016/j.apacoust.2018.07.001. 